\title{
Charge carrier dynamics in CdTe/ZnTe core/shell nanocrystals for photovoltaic applications ${ }^{\S}$
}

\author{
SOURAV MAITI ${ }^{\mathrm{a}}$, PRANAV ANAND ${ }^{\mathrm{a}}$, FARAZUDDIN AZLAN ${ }^{\mathrm{a}}$ and HIRENDRA N GHOSH ${ }^{\mathrm{a}, \mathrm{b}, *}$ \\ ${ }^{a}$ Radiation and Photochemistry Division, Bhabha Atomic Research Centre, Mumbai 400 085, Maharashtra, \\ India \\ ${ }^{\mathrm{b}}$ Institute of Nano Science and Technology, Mohali 160 062, Punjab, India \\ E-mail: hnghosh@inst.ac.in; hnghosh@barc.gov.in
}

MS received 2 August 2018; revised 24 August 2018; accepted 27 August 2018; published online 26 September 2018

\begin{abstract}
CdTe/ZnTe type-II core/shell nanocrystals were synthesized and characterized by the red-shift in the UV-Vis absorption and emission spectra along with the increase in both emission quantum yield and lifetime. The charge carrier dynamics was investigated through ultrafast transient absorption spectroscopy revealing the excited state carrier distribution and the dynamics through which the carriers decay. Upon laser pulse excitation the transient absorption spectrum was characterized by a broad ground state bleach signal in the core/shell nanocrystals in accord with the absorption spectra. Slower electron cooling was found in the core/shell nanocrystals compared to the CdTe core due to the type-II band-alignment that decouples the electron from hole preventing Auger-assisted electron cooling process. The recombination was found to be much slower in the core/shell nanocrystals due to the better surface passivation provided by the ZnTe shell eliminating the surface trapping process. The low band-gap CdTe based nanocrystals with a charge separated state are a viable candidate for photovoltaic applications and finally, we have investigated the potentiality of the synthesized nanocrystals as sensitizers in quantum dot solar cells.
\end{abstract}

Keywords. Ultrafast spectroscopy; solar enery conversion; Quasi type II core shell, electron transfer.

\section{Introduction}

Semiconducting nanocrystals (NCs) or quantum dots (QDs) are fundamentally interesting due to their quantum confined nature and have tremendous application potential in solar cells, light emitting devices, biological imaging, lasers and photocatalysis. ${ }^{1-12}$ In this regard, core/shell NCs offers more flexibility in terms of charge carrier confinement as the charge carrier localization can be tuned by varying the core and shell materials. For example, in the type-I structure both electron and hole are confined in the core whereas in type-II structure the electron and hole are spatially separated due to staggered band alignment between the core and shell. ${ }^{13-15}$ Due to this electron-hole decoupling, the type-II nanocrystal systems have long radiative lifetimes and are better candidates for photovoltaic devices. ${ }^{14,16-19}$

\footnotetext{
*For correspondence

$\S$ Dedicated to Professor M V George on the occasion of his $90^{\text {th }}$ Birth Anniversary.
}

CdTe based NCs with bulk band-gap of $1.7 \mathrm{eV}$ are promising candidates for solar cell applications. ${ }^{20}$ Earlier we have investigated the carrier dynamics in CdTe/ZnTe NCs synthesized in the aqueous phase at low temperatures having a high density of surface trap states. ${ }^{21}$ However, for photovoltaic applications we need high-quality NCs having less trap density. Herein we have investigated the ultrafast exciton dynamics utilizing transient absorption (TA) spectroscopy in $\mathrm{CdTe} / \mathrm{ZnTe}$ core/shell NCs synthesized through hightemperature hot injection method. These NCs have higher quantum yield and photoluminescence lifetime compared to the CdTe core implying their superior optoelectronic properties. In TA experiments, the core/shell NCs showed signatures of charge separation due to the type-II nature where the electron and hole are spatially decoupled from each other. Significantly, the recombination was found to be much slower in the core/shell NCs in accord with the improved surface passivation. As a proof-of-concept, we fabricated sensitized solar cell devices with the NCs synthesized. For CdTe/ZnTe NCs the power conversion efficiency (PCE) was found to be $1.35 \%$ whereas pure CdTe had negligible PCE 
due to instability in polysulfide electrolyte. ${ }^{22}$ Therefore, the improved surface passivation provided by $\mathrm{ZnTe}$ and the charge separated state help to attain better PCE in the core/shell CdTe/ZnTe NCs.

\section{Experimental}

\subsection{Materials}

Cadmium oxide (CdO, 99.5\%), tellurium ( $\mathrm{Te}, 99.99 \%)$, sulfur powder $(99.99 \%)$, zinc acetate $\left(\mathrm{ZnAc}_{2}, 99.9 \%\right)$, octadecene (90\%) oleic acid (90\%), tri-octyl phosphine (TOP, 90\%), cadmium nitrate tetrahydrate $\left(\mathrm{Cd}\left(\mathrm{NO}_{3}\right)_{2} \cdot 4 \mathrm{H}_{2} \mathrm{O}\right)$, sodium sulfide $\left(\mathrm{Na}_{2} \mathrm{~S}\right)$, zinc nitrate hexahydrate $\left(\mathrm{Zn}\left(\mathrm{NO}_{3}\right)_{2} \cdot 6 \mathrm{H}_{2} \mathrm{O}\right)$, potassium chloride $(\mathrm{KCl})$, potassium hydroxide $(\mathrm{KOH})$ and mercaptopropionic acid (MPA) were obtained from SigmaAldrich and used as received. $\mathrm{TiO}_{2}$ transparent (DSL 18NR$\mathrm{T}$ ) and scattering (WER2-O) were purchased from Dyesol. AR grade chloroform, acetone, methanol and de-ionized (DI) water were used for cleaning the nanocrystals.

\subsection{Synthesis of CdTe and CdTe/ZnTe nanocrystals}

CdTe NCs were synthesized through a high-temperature hot injection method as reported earlier. ${ }^{23,24}$ As a summary, $2 \mathrm{mmol}$ of $\mathrm{CdO}, 8 \mathrm{mmol}$ of oleic acid and $10 \mathrm{~mL}$ of 1 octadecene were loaded in a three-necked flask and heated to $260^{\circ} \mathrm{C}$ under nitrogen until the solution becomes colorless indicating the formation of cadmium oleate. The Te-TOP precursor ( $1 \mathrm{mmol})$ in $2 \mathrm{~mL}$ octadecene was rapidly injected into the mixture and the NCs were grown at $240{ }^{\circ} \mathrm{C}$. The reaction mixture was cooled to room temperature and the $\mathrm{CdTe}$ NCs were cleaned with methanol twice and dispersed in chloroform. The ZnTe shell was grown through successive ionic layer adsorption and reaction (SILAR) technique as described previously. ${ }^{23,25}$ Briefly, the shell was grown layer-by-layer (where one monolayer, ML is around $0.32 \mathrm{~nm}$ ) through alternate injection of $\mathrm{Zn}$-oleate and Te-TOP at an interval of 10 min. 2ML of the shell was grown on the CdTe core.

\subsection{Determination of quantum yield}

The photoluminescence quantum yield (PLQY) was determined through a relative method using standard dye as a reference.

$$
\begin{aligned}
Q Y_{\text {Sample }}= & \frac{P L_{\text {sample }}}{P L_{\text {reference }}} \times \frac{A b s_{\text {reference }}}{A b s_{\text {sample }}} \\
& \times\left(\frac{\eta_{\text {reference }}}{\eta_{\text {sample }}}\right)^{2} \times Q Y_{\text {reference }}
\end{aligned}
$$

Here, $\mathrm{PL}_{\text {sample }}$ and $\mathrm{PL}_{\text {reference }}$ are the areas under the emission spectra; $\mathrm{Abs}_{\text {sample }}$ and $\mathrm{Abs}_{\text {reference }}$ are the absorbance at the excitation wavelength $(525 \mathrm{~nm})$ of the sample and reference respectively. $\eta$ is the refractive index of the solvent.
The absorbance was kept below 0.1 during quantum yield measurement.

\subsection{Photoluminescence lifetime measurements}

The photoluminescence lifetime was measured through time-correlated single photon counting (TCSPC) method after exciting the nanocrystals with $445 \mathrm{~nm}$ diode laser in IBH (UK) instrument.

\subsection{Ultrafast transient absorption measurements}

The NCs dispersed in chloroform were excited with $400 \mathrm{~nm}$ pump beam and the excited state dynamics was probed in the visible region. The output $(800 \mathrm{~nm}, 5 \mathrm{~nJ}, 50 \mathrm{fs}, 88 \mathrm{MHz})$ from a mode-locked Ti:sapphire oscillator (Tissa 50, CDP, Moscow, Russia) was amplified in a multipass amplifier to obtain $50 \mathrm{fs}$ pulses with $1 \mathrm{kHz}$ repetition rate having $1 \mathrm{~mJ}$ energy per pulse. The pump beam of $400 \mathrm{~nm}$ was generated from a $\beta$-barium borate (BBO) crystal after frequency doubling. The broadband probe beam was generated after focusing the $800 \mathrm{~nm}$ laser on a sapphire crystal. The number of exciton per NC was kept $<1$ to avoid multi-excitonic effects. The NC sample was kept in a rotating cell to avoid sample damage from the pump laser. The data (spectrum and kinetics) was collected through Excipro software and the multiexponential fitting of the kinetic traces was performed in Lab-view software. Details of the experimental set-up are provided in our earlier publications. ${ }^{26,27}$

\subsection{Solar cell fabrication and characterization}

The quantum dot sensitized solar cells were prepared following reported procedures with required modifications. ${ }^{28-34}$ The process will be described briefly here. The oleic acid capped NCs were phase transferred to the aqueous phase with mercaptopropionic acid for sensitization of the $\mathrm{TiO}_{2}$ photoanodes. The $\mathrm{TiO}_{2}$ photoanodes were prepared through doctor blade technique, first by putting transparent $\mathrm{TiO}_{2}$ followed by baking at $450{ }^{\circ} \mathrm{C}$ for $30 \mathrm{~min}$. Then a scattering $\mathrm{TiO}_{2}$ layer was put through baking at $450{ }^{\circ} \mathrm{C}$ for another $30 \mathrm{~min}$. The MPA-capped NCs were drop-casted on the $\mathrm{TiO}_{2}$ photoanode and kept for $6 \mathrm{~h}$ before washing with de-ionized water. On this 4 layers of $\mathrm{CdS}$ and 2 layers of $\mathrm{ZnS}$ was put through SILAR technique. For counter electrode, $\mathrm{Cu}_{2} \mathrm{Se}$ was utilized. ${ }^{35} \mathrm{The} \mathrm{NC}$ sensitized $\mathrm{TiO}_{2}$ photoanode was combined with the $\mathrm{Cu}_{2} \mathrm{Se}$ counter electrode and polysulfide $\left(\mathrm{S}^{2-} / \mathrm{S}_{\mathrm{n}}^{2-}\right)$ electrolyte and assembled with two binder clips. The current density-voltage $(\mathrm{J}-\mathrm{V})$ curves were obtained under 1 Sun intensity $\left(100 \mathrm{~mW} / \mathrm{cm}^{2}\right)$ in a solar simulator (Peccell, PEC-L01) combined with Keithley 2400 as a source meter. The 1 Sun intensity was confirmed using a standard photodiode. The power conversion efficiency $(\eta)$ was measured as

$\eta=\frac{J_{S C} \times V_{O C} \times F F}{P_{i n}}$ 
where $\mathrm{J}_{\mathrm{SC}}$ is the short circuit current density, $\mathrm{V}_{\mathrm{OC}}$ is the open circuit voltage, FF is the fill factor and $\mathrm{P}_{\text {in }}$ the input power $\left(100 \mathrm{~mW} / \mathrm{cm}^{2}\right)$. The incident photon to current conversion efficiency (IPCE) spectra was obtained from the set up provided by Peccell (PEC-S20).

\section{Results and Discussion}

$\mathrm{CdTe} / Z n T e$ core/shell NCs represent a type-II system due to staggered band alignment between the CdTe core and ZnTe shell. Therefore, the electron will be localized in the CdTe core whereas the hole will be in the ZnTe shell making a spatially decoupled system.

The CdTe NC core synthesized through high-temperature hot injection method as described in the experimental section were of $\sim 4.5 \mathrm{~nm}$ size as determined from the absorption maxima $(\sim 655 \mathrm{~nm}) .{ }^{36}$ The ZnTe shell of $2 \mathrm{ML}$ was grown through SILAR technique. Figure 1 depicts the UV-vis absorption spectra of both the CdTe core and CdTe/ZnTe core/shell NCs where both the absorption and photoluminescence (PL) maxima has red-shifted after deposition of the shell indicating the formation of the core/shell structure. The broad absorption spectra with indistinct excitonic features also indicate type-II structure formation. ${ }^{13,17}$ The PL quantum yield has increased from $6 \%$ to $20 \%$ in accord with the PL lifetime which increased from 21 ns to 28.4 ns upon ZnTe shell deposition. For CdTe, the PL lifetime was bi-exponential with 2.34 ns (27\%) and 28 ns $(73 \%)$ components. The first component is due to radiative recombination whereas the second component is due to shallow trap mediated recombination process. ${ }^{25,37}$ In the core/shell NCs the bi-exponential PL decay components are $3.4 \mathrm{~ns}(22 \%)$ and $35.3 \mathrm{~ns}$ (78\%), respectively. The radiative component has a longer lifetime implying better surface passivation provided by the ZnTe shell.

Figure 1(B) shows the transmission electron micrographs (TEM) of CdTe/ZnTe NCs. The size of the core/shell NCs is $\sim 5.78 \mathrm{~nm}$ which is close to $2 \mathrm{ML}$ of ZnTe shell growth.

To ascertain the effect of better passivation and charge separation we have performed ultrafast transient absorption measurements after exciting the NCs with $400 \mathrm{~nm}$ pump and monitoring the excited state dynamics throughout the visible range. In the case of CdTe, the transient absorption spectra are characterized by a bleach centered around $655 \mathrm{~nm}$ which can be attributed to the ground state $1 \mathrm{~S}$ bleach $\left(1 \mathrm{~S}_{\mathrm{e}}-1 \mathrm{~S}_{3 / 2}\right)$ as shown in Figure 2. The bleach signal can have a contribution from both electron and hole. However, for II-VI semiconductors the contribution of the hole can be neglected due to the high density of hole states as the effective mass of a hole is much higher than the electron. ${ }^{38}$ Therefore, the bleach signal provides information about the electron dynamics. ${ }^{39}$

As we are exciting the $\mathrm{NC}$ with $400 \mathrm{~nm}$ laser which is higher in energy than the $1 \mathrm{~S}$ transition in CdTe hot electron is created in the conduction band. This hot electron circumvents the phonon-bottleneck through an Augerassisted mechanism by transferring its excess energy to valence band hole. ${ }^{40,41}$ This electron cooling process can be identified from the $1 \mathrm{~S}$ bleach growth which signifies
A

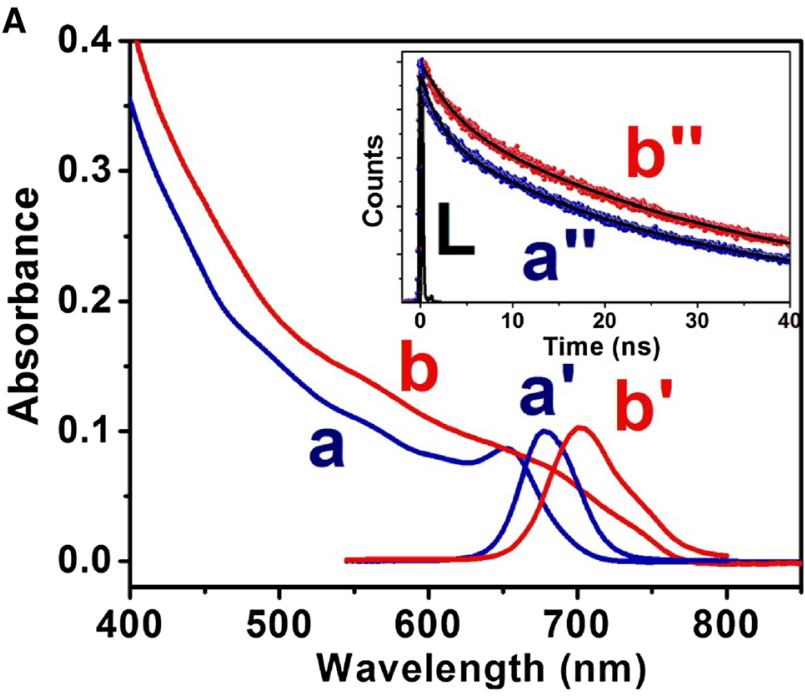

B

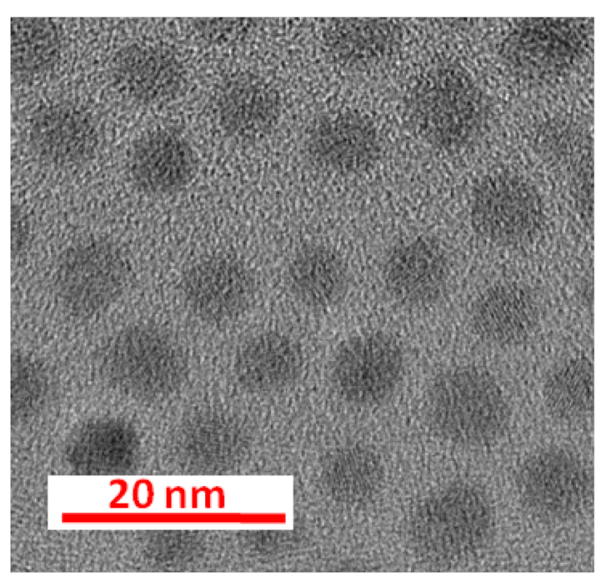

Figure 1. (A) UV-Vis absorption spectra (a, b) and photoluminescence spectra (a', b') of (a, a') CdTe and (b, b') CdTe/ZnTe NCs. (Inset) Photoluminescence decay traces of (a") CdTe and (b") CdTe/ZnSe NCs. L denotes the lamp profile. (B) Transmission electron micrograph of CdTe/ZnTe nanocrystals. 


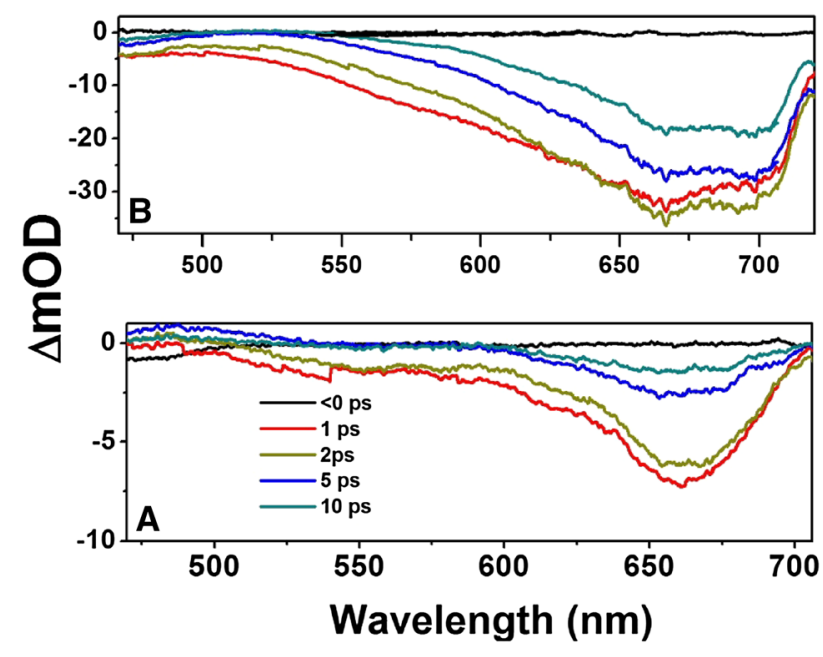

Figure 2. Transient absorption spectra of (A) CdTe and (B) $\mathrm{CdTe} / \mathrm{ZnSe}$ NCs upon $400 \mathrm{~nm}$ pump excitation.

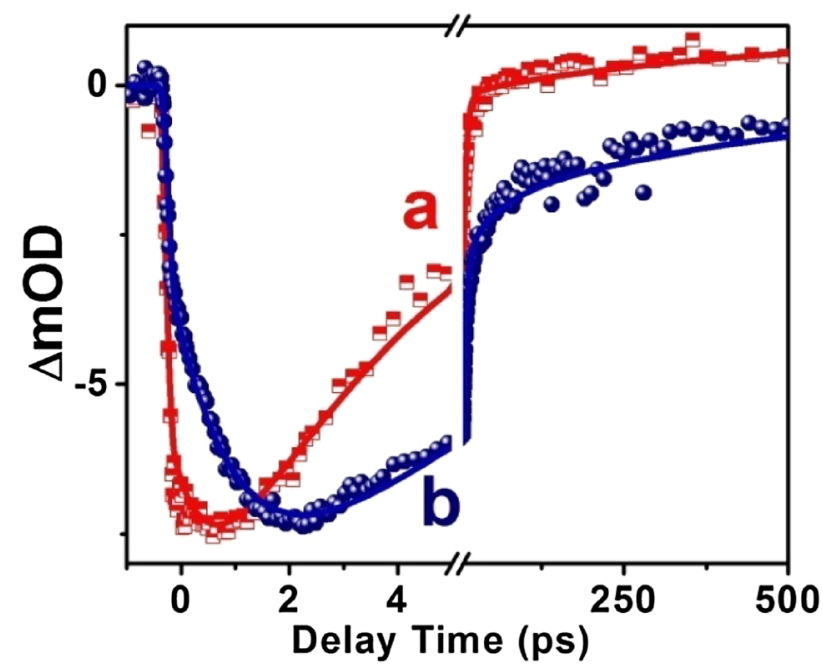

Figure 3. Ground state bleach recovery kinetics of (a) CdTe and (b) CdTe/ZnTe NCs. The solid lines are fitted data.

population of the $1 \mathrm{~S}$ state. The subsequent depopulation through trapping and recombination can be interpreted from bleach recovery kinetics. ${ }^{42}$ The ground state bleach recovery kinetics of the NCs have been compared in Figure 3. For CdTe, the bleach growth has a pulse width limited $<100 \mathrm{ps}(75 \%)$ and $0.65 \mathrm{ps}(25 \%)$ growth component as well as 5 ps (85\%) and 50 ps (15\%) recovery components. The 0.65 ps component can be attributed to electron cooling to the $1 \mathrm{~S}_{\mathrm{e}}$ state. ${ }^{23,43,44}$

For the $\mathrm{CdTe} / \mathrm{ZnTe} \mathrm{NCs}$ there is broad bleach starting from $650 \mathrm{~nm}$ as depicted in figure 2. However, due to stimulated emission from the $\mathrm{NC}$, the TA spectral shape is obscured beyond $720 \mathrm{~nm}$. Here, the bleach growth dynamics can be fitted with a pulse width limited $<100$ fs $(45 \%)$ and $1.6 \mathrm{ps}(55 \%)$. Therefore, we observe much slower bleach growth implying slower
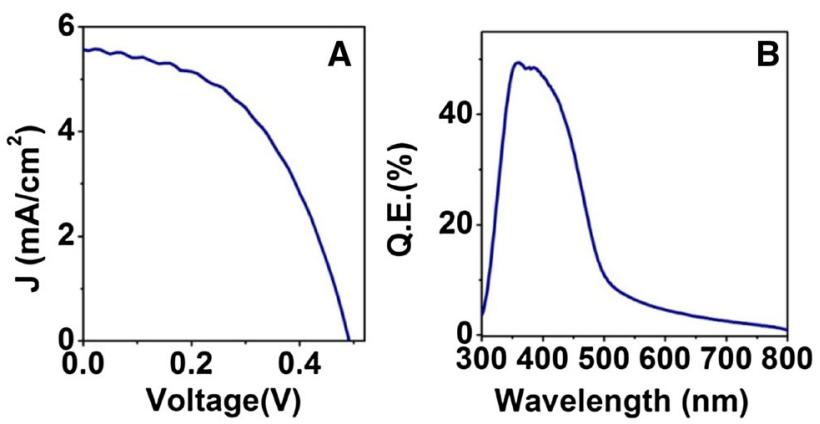

Figure 4. (A) Current density (J-V) and (B) IPCE spectrum of CdTe/ZnTe NCs.

electron cooling compared to pure CdTe NCs. This can be attributed to electron-hole decoupling due to the type-II nature of the system. The Auger-assisted energy transfer depends on the electron-hole wave function overlap. ${ }^{40,41}$ In CdTe/ZnTe NCs the hole moves from the $\mathrm{CdTe}$ core to the $\mathrm{ZnTe}$ shell due to the staggered band alignment decreasing the overlap. On the other hand, the bleach recovery components can be fitted with 4.5 ps (60\%), 50 ps (30\%) and $>400$ ps (10\%). Therefore, the excitons are longer lived in the core/shell NCs due to the surface passivation provided by the $\mathrm{ZnTe}$ shell.

We have tested the potentiality of the core/shell $\mathrm{CdTe} / \mathrm{ZnTe} \mathrm{NCs}$ as sensitizer material in quantum dot solar cells. The details of the device fabrication protocol have been provided in the experimental section. The NC sensitized $\mathrm{TiO}_{2}$ photoanodes were assembled with the $\mathrm{Cu}_{2} \mathrm{Se}$ counter electrode and polysulfide electrolyte for solar cell characterization through J-V and IPCE measurements. The CdTe NCs have a tendency to degrade in presence of polysulfide electrolyte and the PCE was found to be negligible. On the other hand, for $\mathrm{CdTe} / \mathrm{ZnTe}$ NCs, we have obtained $1.35 \pm 0.05 \%$ PCE with short circuit current $\left(\mathrm{J}_{\mathrm{SC}}\right) 5.56 \mathrm{~mA} / \mathrm{cm}^{2}$, open circuit voltage $\left(\mathrm{V}_{\mathrm{OC}}\right) 0.49 \mathrm{~V}$ and fill factor $(\mathrm{FF}) 49 \%$ as represented in Figure 4. The IPCE spectra also show broad photoresponse up to $750 \mathrm{~nm}$ in accord with absorption spectra. This implies that the core/shell NCs are stable in presence of the polysulfide electrolyte due to surface passivation provided by the $\mathrm{ZnTe}$ shell. Moreover, the extended absorption and charge separation due to typeII structure makes CdTe/ZnTe as a promising material for solar cell applications.

\section{Conclusions}

CdTe/ZnTe core/shell NCs having type-II band alignment were synthesized through high-temperature colloidal method. The steady state absorption and emission are red shifted in the core/shell structure with an 
increase in both the PLQY and PL lifetime due to the formation of the type-II structure. The ultrafast transient absorption spectroscopic measurements revealed signatures of charge separation as the electron cooling slowed down due to the electron-hole decoupling. The recombination was also slower in the core/shell NC depicting the importance of surface passivation by ZnTe. Finally, CdTe/ZnTe NCs-sensitized solar cell devices were fabricated with $1.35 \%$ efficiency and a broad photo-response in the IPCE spectra attracting further research to optimize the photovoltaic performance.

\section{Acknowledgements}

S.M. acknowledges CSIR for a research fellowship. This work was supported by "DAE-SRC Outstanding Research Investigator Award" (Project/Scheme No.: DAE-SRC/2012/ 21/13-BRNS) granted to H.N.G.

\section{References}

1. Alivisatos A P 1996 Semiconductor clusters, nanocrystals, and quantum dots Science 271933

2. Bruchez M, Moronne M, Gin P, Weiss S and Alivisatos A P 1998 Semiconductor nanocrystals as fluorescent biological labels Science 2812013

3. Carey G H, Abdelhady A L, Ning Z, Thon S M, Bakr O M and Sargent E H 2015 Colloidal quantum dot solar cells Chem. Rev. 11512732

4. Semonin O E, Luther J M and Beard M C 2012 Quantum dots for next-generation photovoltaics Mater. Today $\mathbf{1 5}$ 508

5. Talapin D V, Lee J-S, Kovalenko M V and Shevchenko E V 2009 Prospects of colloidal nanocrystals for electronic and optoelectronic applications Chem. Rev. 110389

6. Kamat P V 2013 Quantum dot solar cells. The next big thing in photovoltaics J. Phys. Chem. Lett. 4908

7. Singhal P and Ghosh H N 2018 Hot charge carrier extraction from semiconductor quantum dots J. Phys. Chem. C 12217586

8. Ghosh H N, Maiti S, Dana J and Tripathi V S 2018 Direct correlation of excitonics with efficiency in core shell quantum dot solar cell Chem. Eur. J. 242418

9. Shojaei A F, Tabatabaeian K, Zanjanchi M A, Moafi H F and Modirpanah N 2015 Synthesis, characterization and study of catalytic activity of silver doped $\mathrm{ZnO}$ nanocomposite as an efficient catalyst for selective oxidation of benzyl alcohol J. Chem. Sci. 127481

10. Kandasamy K, Singh H B and Kulshreshtha S K 2009 Synthesis and characterization of CdS and CdSe nanoparticles prepared from novel intramolecularly stabilized single-source precursors J. Chem. Sci. 121293

11. Datta K K R, Srinivasan B, Balaram H and Eswaramoorthy M 2008 Synthesis of agarose-metal/semiconductor nanoparticles having superior bacteriocidal activity and their simple conversion to metal-carbon composites $J$. Chem. Sci. 120579
12. Nasr C, Hotchandani $\mathrm{S}$ and Kamat P V 1995 $\mathrm{CdSe}-\mathrm{SnO} 2$ coupled semiconductor thin films: Electrochemical and photoelectrochemical studies Proc. Ind. Acad. Sci. Chem. Sci. 107691

13. Kim S, Fisher B, Eisler H-J and Bawendi M 2003 Type-II Quantum dots: CdTe/CdSe (core/shell) and CdSe/ZnTe (core/shell) heterostructures J. Am. Chem. Soc. 125 11466

14. Lo S S, Mirkovic T, Chuang C-H, Burda C and Scholes G D 2011 Emergent properties resulting from type-II band alignment in semiconductor nanoheterostructures $A d v$. Mater. 23180

15. Maiti S, Debnath T and Ghosh H N 2016 Elucidating the electronic cross-talk dynamics across the heterointerface of Janus CdSe/PbSe nanocrystals J. Phys. Chem. C 120 29054

16. Reiss P, Protière M and Li L 2009 Core/shell semiconductor nanocrystals Small 5154

17. Smith A M, Mohs A M and Nie S 2009 Tuning the optical and electronic properties of colloidal nanocrystals by lattice strain Nat. Nanotechnol. 456

18. Smith A M and Nie S 2010 Semiconductor nanocrystals: Structure, properties, and band gap engineering Acc. Chem. Res. 43190

19. Kaniyankandy S, Rawalekar S, Verma S and Ghosh H N 2011 Ultrafast hole transfer in CdSe/ZnTe type II coreshell nanostructure J. Phys. Chem. C 1151428

20. Yang J and Zhong X 2016 CdTe based quantum dot sensitized solar cells with efficiency exceeding $7 \%$ fabricated from quantum dots prepared in aqueous media J. Mat. Chem. A 416553

21. Rawalekar S, Kaniyankandy S, Verma S and Ghosh H N 2011 Effect of surface states on charge-transfer dynamics in type II CdTe/ZnTe Core-shell quantum dots: A femtosecond transient absorption study J. Phys. Chem. C 11512335

22. Bang J H and Kamat P V 2009 Quantum dot sensitized solar cells. A tale of two semiconductor nanocrystals: CdSe and CdTe ACS Nano 31467

23. Maiti S, Debnath T, Maity P and Ghosh H N 2015 Latticestrain-induced slow electron cooling due to quasi-type-II behavior in type-I CdTe/ZnS nanocrystals J. Phys. Chem. C 1198410

24. Yu W W, Wang Y A and Peng X 2003 Formation and stability of size-, shape-, and structure-controlled CdTe nanocrystals: Ligand effects on monomers and nanocrystals Chem. Mater. 154300

25. Maiti S, Debnath T, Maity P and Ghosh H N 2016 Tuning the charge carrier dynamics via interfacial alloying in core/shell CdTe/ZnSe NCs J. Phys. Chem. C 1201918

26. Maity P, Debnath T, Chopra U and Ghosh H N 2015 Cascading electron and hole transfer dynamics in $\mathrm{CdS} / \mathrm{CdTe}$ core-shell sensitized bromo-pyrogallol red (Br-PGR): Slow charge recombination in type II regime Nanoscale 7 2698

27. Debnath T, Maity P, Maiti S and Ghosh H N 2014 Electron trap to electron storage center in specially aligned Mn-doped CdSe d-Dot: A step forward in the design of higher efficient quantum-dot solar cell J. Phys. Chem. Lett. 52836

28. Pan Z, Zhao K, Wang J, Zhang H, Feng $Y$ and Zhong $\mathrm{X} 2013$ Near infrared absorption of $\mathrm{CdSe}_{\mathrm{X}} \mathrm{Te}_{1-\mathrm{x}}$ alloyed 
quantum dot sensitized solar cells with more than $6 \%$ efficiency and high stability ACS Nano 75215

29. Liu L and Zhong X 2012 A General and reversible phase transfer strategy enabling nucleotides modified highquality water-soluble nanocrystals Chem. Commun. 48 5718

30. Maiti S, Azlan F, Anand P, Jadhav Y, Dana J, Haram S K and Ghosh H N 2018 Boosting the efficiency of quantum dot-sensitized solar cells through formation of the cationexchanged hole transporting layer Langmuir 3450

31. Debnath T, Parui K, Maiti S and Ghosh H N 2017 An Insight into the interface through excited-state carrier dynamics for promising enhancement of power conversion efficiency in a Mn-doped $\mathrm{CdZnSSe}$ gradient alloy Chem. Eur. J. 233755

32. Maity P, Maiti S, Debnath T, Dana J, Guin S K and Ghosh H N 2016 Intraband electron cooling mediated unprecedented photocurrent conversion efficiency of $\mathrm{CdS}_{\mathrm{X}} \mathrm{Se}_{1-\mathrm{x}}$ alloy QDs: Direct correlation between electron cooling and efficiency J. Phys. Chem. C 12021309

33. Dana J, Maiti S, Tripathi V S and Ghosh H N 2018 Direct correlation of excitonics with efficiency in a core-shell quantum dot solar cell Chem. Eur. J. 242418

34. Dana J, Anand P, Maiti S, Azlan F, Jadhav Y, Haram S K and Ghosh H N 2018 Inhibiting interfacial charge recombination for boosting power conversion efficiency in $\mathrm{CdSeAu}$ nanohybrid sensitized solar cell J. Phys. Chem. C 12213277

35. Zhang H, Wang C, Peng W, Yang C and Zhong X 2016 Quantum dot sensitized solar cells with efficiency up to
$8.7 \%$ based on heavily copper-deficient copper selenide counter electrode Nano Energy 2360

36. Yu W W, Qu L, Guo W and Peng X 2003 Experimental determination of the extinction coefficient of CdTe, CdSe, and CdS nanocrystals Chem. Mater. 152854

37. Fitzmorris B C, Cooper J K, Edberg J, Gul S, Guo J and Zhang J Z 2012 Synthesis and structural, optical, and dynamic properties of core/shell/shell CdSe/ZnSe/ZnS quantum dots J. Phys. Chem. C 11625065

38. Klimov V I 2000 Optical nonlinearities and ultrafast carrier dynamics in semiconductor nanocrystals J. Phys. Chem. B 1046112

39. Sewall S, Cooney R, Anderson K, Dias E and Kambhampati P 2006 State-to-state exciton dynamics in semiconductor quantum dots Phys. Rev. B 74235328

40. Efros A L, Kharchenko V A and Rosen M 1995 Breaking the phonon bottleneck in nanometer quantum dots: Role of Auger-like processes Solid State Commun. 93281

41. Kambhampati P 2011 Unraveling the structure and dynamics of excitons in semiconductor quantum dots Acc. Chem. Res. 441

42. Kaniyankandy S, Rawalekar S, Verma S, Palit D K and Ghosh H N 2010 Charge carrier dynamics in thiol capped CdTe quantum dots Phys. Chem. Chem. Phys. 124210

43. Klimov V, Mikhailovsky A, McBranch D, Leatherdale C and Bawendi M 2000 Mechanisms for intraband energy relaxation in semiconductor quantum dots: The role of electron-hole interactions Phys. Rev. B 61 R13349

44. Pandey A and Guyot-Sionnest P 2008 Slow electron cooling in colloidal quantum dots Science $\mathbf{3 2 2} 929$ 